\title{
Optimization, Biopharmaceutical Profile and Therapeutic Efficacy of Pioglitazone-loaded PLGA-PEG Nanospheres as a Novel Strategy for Ocular Inflammatory Disorders
}

\begin{abstract}
Authors: Marcelle Silva-Abreu ${ }^{1,2}$, Ana Cristina Calpena ${ }^{1,2}$, Marta Espina ${ }^{1,2}$, Amelia M. Silva ${ }^{3,4}$, Alvaro Gimeno $^{5}$, María Antonia Egea ${ }^{1,2}$, María Luisa García ${ }^{1,2}$

${ }^{1}$ Department of Pharmacy, Pharmaceutical Technology and Physical Chemistry, Faculty of Pharmacy and Food Sciences, University of Barcelona, Barcelona 08028, Spain.

${ }^{2}$ Institute of Nanoscience and Nanotechnology (IN2UB). University of Barcelona, Barcelona, Spain.

${ }^{3}$ Department of Biology and Environment, School of Life and Environmental Sciences, (ECVA, UTAD), University of Trás-os-Montes and Alto Douro, Quinta de Prados, Vila Real 5001-801, Portugal.

${ }^{4}$ Centre for the Research and Technology of Agro-Environmental and Biological Sciences, University of Trás-os-Montes and Alto Douro, CITAB-UTAD, Vila-Real 5001-801, Portugal.

${ }^{5}$ Animal Facility, Bellvitge Health Sciences Campus, University of Barcelona, Barcelona 08907, Spain.
\end{abstract}

Corresponding author: María Luisa García. Department of Pharmacy, Pharmaceutical Technology and Physical Chemistry, Faculty of Pharmacy and Food Sciences, University of Barcelona, Barcelona 08028, Spain. Phone: +34 9340245 52. E-mail: rdcm@ ub.edu

\section{ABSTRACT}

Purpose The main goal of this study was to encapsulate Pioglitazone (PGZ), in biodegradable polymeric nanoparticles as a new strategy for the treatment of ocular inflammatory processes.

Methods To improve their biopharmaceutical profile for the treatment of ocular inflammatory disorders, nanospheres (NSs) of PGZ were formulated by factorial design with poly (lactic-co-glycolic acid) polyethylene glycol (PLGA-PEG). Interactions drugpolymer have been carried out by spectroscopic (X-ray spectroscopy, FTIR) and thermal methods (DSC). The PGZ-NSs were tested for their in vitro release profile, cytotoxicity, and ocular tolerance (HET-CAM test); ex vivo corneal permeation, and in vivo inflammatory prevention and bioavailability.

Results The optimized system showed a negative surface charge of $-13.9 \mathrm{mV}$, an average particle size $\left(\mathrm{Z}_{\mathrm{av}}\right)$ of around $160 \mathrm{~nm}$, a polydispersity index (PI) below 0.1 , and a high encapsulation efficiency (EE) of around 92\%. According to the DSC results, the drug was incorporated into the NSs polymeric matrix. The drug release was sustained for up to 14 hours. PGZ-NSs up to $10 \mu \mathrm{g} / \mathrm{ml}$ exhibited no retinoblastoma cell toxicity. The ex vivo 
corneal and scleral permeation profiles of PGZ-NSs showed that retention and permeation through the sclera were higher than through the cornea. Ocular tolerance in vitro and in vivo demonstrated the non-irritant character of the formulation.

Conclusion The in vivo anti-inflammatory efficacy of developed PGZ-NSs indicates this colloidal system could constitute a new approach to prevent ocular inflammation.

KEY WORDS: Pioglitazone; nanospheres; ocular anti-inflammatory efficacy; PLGAPEG; drug delivery.

\section{Abbreviations}

PGZ: Pioglitazone, NSs: nanospheres; PPAR $\gamma$ : peroxisome proliferator-activated receptor, TEM: transmission electron microscopy; PI: polydispersity index; ZP: Zeta potential; PLGA: polylactic-co-glycolic acid; PEG: polyethylene glycol; DSC: Differential Scanning Calorimetry

\section{INTRODUCTION}

Pioglitazone (PGZ) (5-[[4-[2-(5-ethylpyridin-2-yl)ethoxy]phenyl]methyl]-1,3thiazolidine-2,4-diona) belongs to the class of the thiazolidinediones (TZDs) for clinical treatment of type 2 diabetes. This drug is an agonist of the peroxisome proliferatoractivated receptor (PPAR $\gamma$ ) it is significantly important in the regulation of the immune and inflammatory responses. The anti-inflammatory effects are induced as a response to a negative regulation of macrophage activation and differentiation. Previous studies into PPAR $\gamma$ receptors have reported functions such as: anti-inflammatory, anti-angiogenic, antifibrotic, anti-tumor effects, and neuroprotection. Moreover, it has a protective effect on inflammatory ocular process (1-4). 
PGZ has the capacity to inhibit the local development of several inflammatory factors: these included IFN- $\gamma$ and IL-6 in vivo. This effect was made clear in the measurement of intraocular cytokines and chemokines (3). The activation of PPAR $\gamma$ may suppress the production of inflammatory factors. This may work through the direct inhibition of their production by intraocular monocytes or T cells. PGZ may also operate by inhibiting corneal fibroblast migration, thus cutting down on corneal fibroblast-induced collagen contraction as the cornea heals (1). In ocular therapies, one of the difficulties is for an adequate drug concentration to be delivered to the site of action and sustained. Normally, in the cases when ophthalmic formulations are used, less than $5 \%$ of the applied drug actually permeates the cornea and arrives to the intraocular tissues (5). This problematic performance means that it is necessary to instill the formulation several times a day in order to achieve the therapeutic efficacy, which is often associated with adverse effects. When the drug is retained in the pre-corneal area and the penetration is through to the cornea, this is of great benefit to the ophthalmic therapy (5).

In past studies it is has been indicated that controlled release systems have an interesting potential for ocular drug delivery (6-10). These systems may intensify the ocular retention through prolonging it. Therefore, different ocular distributions in colloidal carriers greatly aid in achieving satisfactory treatment of eye pathologies, with the possibility of targeting different eye regions (11). Liposomes and polymeric nanoparticles (NPs) including nanospheres (NSs) and nanocapsules, are among the most successful approaches for ocular delivery systems. Numerous studies have reported on the effectiveness of encapsulation drugs with biodegradable polymers to treat ocular disorders. This is because of their biocompatibility and biodegradability, and they show an appropriate drug delivery in different tissues of the eye $(6,10,12-13)$. 
Much attention has been focused on polymeric NPs as potential therapeutic delivery systems. Biodegradable polymers, including polylactic-co-glycolic acid (PLGA), (approved by European Medicine Agency (EMA) and Food and Drug Administration (FDA)) used in various drug delivery systems in humans, exhibit good biocompatibility, small and non-toxic particles can be produced (12). All the foregoing renders them optimal carriers for entrapping biologically active macromolecular drugs, such as polypeptides, proteins, nucleic acids, and vaccine vectors (5). Therefore, they could be a valid alternative for the delivery of drugs into the eye. The addition of a second ingredient such as Polyethylene glycol (PEG) may make them more versatile in terms of the encapsulation and delivery of proteins, and more susceptible to interact with the biological surface (14). Thus, studies have shown that PEG coated NPs have crucial therapeutic potential ensuring a more controlled release of drugs. The PEG associated with the surface of PLGA-NPs creates a hydrophilic coating at the hydrophobic NPs surface, with the advantage that these systems are working their (beneficial) effect over a greater time period, thus avoiding being recognized by the reticuloendothelial system (RES). PEG coating polyester also facilitates the transport of the NPs across the corneal epithelium (15).

The aim of this study was to develop a new ocular delivery system of PGZ loaded PLGAPEG NSs determining which factors influence the physicochemical properties of the particles and drug entrapment efficiency (EE). The NSs were optimized by a factorial design.

The in vitro release profile, ex vivo transcorneal and transcleral permeations and in vivo assays were performed to demonstrate that pioglitazone nanospheres (PGZ-NSs) are suitable for the prevention of ocular inflammatory process. Assays of cellular toxicity, HET-CAM and Draize test were carried out to confirm that these systems do not induced 
eye irritation and they could present novel targets so as to act more effectively against ocular inflammatory diseases.

\section{EXPERIMENTAL}

\section{Materials}

The PGZ was purchased from Capot Chemical (Hangzhou, P.R. China) and Diblock copolymer PLGA-PEG 5\% (50:50) Resomer $^{\circledR}$ was obtained from Evonik Corporation (Birmingham, USA). Tween (Tw) 80 and acetone were purchased from Sigma-Aldrich (Madrid, Spain) and Fisher Scientific (Pittsburgh, USA), respectively. The dialysis membrane MWCO 12,000-14,000 Da. was obtained from Medicell International Ltd. (London, UK). Fertilized hens' eggs were obtained from the GALLSA farm (Tarragona, Spain). Reagents for cell culture were obtained from Gibco (Alfagene, Portugal). The Y79 was acquired from Cell Lines Service (CLS, Eppelheim, Germany) and Alamar Blue from Invitrogen Alfagene ${ }^{\circledR}$ (Portugal) was used in order to estimate the cell viability. Water filtered through a Millipore MilliQ system was used throughout all the experiments. All reagents were up to analytical grade.

\section{Methods}

\section{Optimization and characterization of PGZ-NSs}

PGZ-NSs were obtained by the solvent displacement technique described in accordance with Fessi (16). This technique consists of dissolving the polymer and the compound in an organic solvent, it being a successful method to deliver the lipophilic drug (13).

PGZ was previously solubilized in dimethyl sulfoxide (DMSO) (17), then the PLGAPEG was dissolved in $5 \mathrm{ml}$ of acetone. Once completely dissolved, they were then mixed together. This organic phase was added drop by drop, gently mixing into $10 \mathrm{ml}$ of an aqueous solution of Tw 80 (2\%), used as a surfactant to achieve pH 4.5. DMSO, and 
acetone were evaporated. The NSs dispersion was concentrated to $10 \mathrm{ml}$ under reduced pressure (Bücchi B-480, Flawil, Switzerland).

The factorial design was set up so as to optimize in that the PGZ-NSs needed as few experiments to be carried out as possible. A five-levels central rotatable composite design $2^{3}+$ star was selected to study the main effects and interactions of three independent variables (PGZ, PLGA-PEG and Tw 80 concentrations), on four dependent variables (average particle size $\left(\mathrm{Z}_{\mathrm{av}}\right)$, polydispersity index $(\mathrm{PI})$, zeta potential $(\mathrm{ZP})$ and $\left.\mathrm{EE}\right)$. A pH of 4.5 was kept constant for all the assays. A total of 16 experiments ( 8 factorial points, 6 axial points and 2 replicated center points) to estimate the pure error sum of squares were required using Statgraphics Plus 5.1 software (Table I). The individual influences and the interactions of the three independent variables led to the results and responses observed in the experiments, and following on from this the full second-order polynomial equation below was used to model them (Eq. 1):

$Y=\beta_{0}+\beta_{1} X_{1}+\beta_{2} X_{2}+\beta_{3} X_{3}+\beta_{11} X_{1}^{2}+\beta_{22} X_{2}^{2}+\beta_{33} X_{3}^{2}+\beta_{12} X_{1} X_{2}+\beta_{13} X_{1} X_{3}+\beta_{23} X_{2} X_{3}$ (1)

where $Y$ is the measured response, $\beta_{0}$ to $\beta_{23}$ are the regression coefficients and $\mathrm{X}_{1}, \mathrm{X}_{2}$ and $\mathrm{X}_{3}$ are the three independent variables. To identify the significance of the effects and interactions between them, analysis of variance (ANOVA) was performed for each parameter.

Morphology of PGZ-NSs dispersions was analyzed by transmission electronic microscopy (TEM). Prior to negative staining, UV light was used to activate copper grids and samples were placed on the grid surface. Samples were diluted (1:3) placed in the grids and negative stain was employed with a $2 \%(\mathrm{v} / \mathrm{v})$ uranyl acetate solution. They were 
then dried at room temperature and the samples were examined by TEM on a Jeol 1010 (Tec-nai Spirit TEM, FEI) at $80 \mathrm{kV}$.

Morphometry ( $\mathrm{Z}_{\mathrm{av}}$ and PI) of NSs was determined by photon correlation spectroscopy (PCS) (after 1:10 dilution) with a Zetasizer Nano ZS (Malvern Instruments, Malvern, $\mathrm{UK}$ ) at $25^{\circ} \mathrm{C}$, in a disposable quartz cells (Malvern Instruments).

Surface charge of developed NSs, measured as ZP, was determined by laser-Doppler electrophoresis with the M3 PALS system in Zetasizer Nano ZS. ZP measurements can give information about the possibility of particles aggregation. A greater ZP (in absolute value) results in there being less aggregation coming from repulsion forces between the particles. To calculate this, the Henry equation was used [18], according to (Eq. 2):

$$
\mu_{E}=\frac{\varepsilon Z P f(K a)}{6 \pi \eta}
$$

where $\mu_{\mathrm{E}}$ is the electrophoretic mobility, $\varepsilon$ is the dielectric constant of the medium, ZP is the Zeta potential, $\eta$ is the viscosity of the medium, $\mathrm{K}$ is the Deybye-Hückel parameter and $\mathrm{f}(\mathrm{Ka})$ is a correction factor fully adjusted for the thickness of the electrical double layer $(1 / \mathrm{K})$ and the particle diameter $(\mathrm{a})$. The reported values are the average $\pm \mathrm{SD}$ of at least three different formulation batches (19).

To indirectly determine the EE of PGZ-NSs the concentration of the free drug in the dispersion medium has been determined previous filtration/centrifugation technique (1:10 dilution) by using Ultracell-100K (Amicon ${ }^{\circledR}$ Ultra; Millipore Corporation, Billerica, Massachusetts) centrifugal filter devices at 12,000 rpm for 15 minutes. The EE was made clear as in (Eq. 3): 
$E E(\%)=\frac{\text { Total amount of } P G Z-\text { Free amount of } P G Z}{\text { Total amount of } P G Z} \cdot 100$

High performance liquid chromatography (HPLC) was used in the evaluation of the samples. The mobile phase was : acetonitrile, ammonia acetate $0.1 \mathrm{M}$ and glacial acetic acid, with a flux of $0.7 \mathrm{ml} / \mathrm{min}$ and a volume of injection of $10 \mu \mathrm{l}$. The reported values are the average $\pm \mathrm{SD}$, there being at least three different batches of the formulation. 
Table I. Coded and values of the three experimental factors according to the matrix designed by $2^{3}+$ star central composite rotable factorial design and measured responses.

\begin{tabular}{|c|c|c|c|c|c|c|c|c|c|c|}
\hline \multirow{2}{*}{$\begin{array}{l}\text { Factorial } \\
\text { points }\end{array}$} & \multicolumn{3}{|c|}{$\mathrm{C}_{\mathrm{PGZ}}$} & $\mathrm{C}_{\text {PLGA-PEG }}$ & \multicolumn{3}{|c|}{$\mathrm{C}_{\mathrm{TW} 80}$} & \multicolumn{2}{|c|}{ Measured response } & \multirow[b]{2}{*}{$\mathrm{EE}(\%)$} \\
\hline & Coded level & $(\mathrm{mg} / \mathrm{ml})$ & Coded level & $(\mathrm{mg})$ & Coded level & $(\%)$ & $\mathrm{Z}_{\mathrm{av}}(\mathrm{nm})$ & PI & $\mathrm{ZP}(\mathrm{mV})$ & \\
\hline F1 & -1 & 0.8 & -1 & 90 & -1 & 1.5 & $152.1 \pm 2.4$ & $0.11 \pm 0.01$ & $-13.9 \pm 0.4$ & $93.97 \pm 0.22$ \\
\hline F2 & 1 & 1.2 & -1 & 90 & -1 & 1.5 & $152.9 \pm 1.1$ & $0.09 \pm 0.01$ & $-10.5 \pm 0.1$ & $85.82 \pm 1.56$ \\
\hline F3 & -1 & 0.8 & 1 & 100 & -1 & 1.5 & $165.1 \pm 2.6$ & $0.16 \pm 0.17$ & $-13.0 \pm 0.3$ & $91.66 \pm 2.13$ \\
\hline F4 & 1 & 1.2 & 1 & 100 & -1 & 1.5 & $158.4 \pm 0.8$ & $0.12 \pm 0.03$ & $-10.9 \pm 0.1$ & $84.87 \pm 2.31$ \\
\hline F5 & -1 & 0.8 & -1 & 90 & 1 & 2.5 & $151.0 \pm 1.1$ & $0.11 \pm 0.01$ & $-12.8 \pm 0.3$ & $91.15 \pm 1.54$ \\
\hline F6 & 1 & 1.2 & -1 & 90 & 1 & 2.5 & $152.4 \pm 0.8$ & $0.10 \pm 0.01$ & $-10.7 \pm 0.1$ & $82.01 \pm 0.21$ \\
\hline F7 & -1 & 0.8 & 1 & 100 & 1 & 2.5 & $165.4 \pm 1.7$ & $0.12 \pm 0.02$ & $-13.3 \pm 0.3$ & $94.47 \pm 0.17$ \\
\hline F8 & 1 & 1.2 & 1 & 100 & 1 & 2.5 & $148.9 \pm 2.3$ & $0.10 \pm 0.03$ & $-10.9 \pm 0.4$ & $81.19 \pm 2.32$ \\
\hline F9 & 1.68 & 1.34 & 0 & 95 & 0 & 2.0 & $161.5 \pm 0.6$ & $0.13 \pm 0.01$ & $-10.1 \pm 0.4$ & $80.45 \pm 0.17$ \\
\hline F10 & -1.68 & 0.66 & 0 & 95 & 0 & 2.0 & $148.9 \pm 0.8$ & $0.12 \pm 0.02$ & $-15.6 \pm 0.5$ & $92.89 \pm 1.23$ \\
\hline F11 & 0 & 1 & 1.68 & 103.4 & 0 & 2.0 & $152.5 \pm 0.1$ & $0.12 \pm 0.00$ & $-12.4 \pm 0.2$ & $88.24 \pm 2.42$ \\
\hline F12 & 0 & 1 & -1.68 & 86.6 & 0 & 2.0 & $162.1 \pm 0.4$ & $0.12 \pm 0.03$ & $-12.5 \pm 0.4$ & $93.95 \pm 1.32$ \\
\hline F13 & 0 & 1 & 0 & 95 & 1.68 & 2.84 & $165.0 \pm 0.3$ & $0.17 \pm 0.01$ & $-12.8 \pm 0.2$ & $87.94 \pm 1.12$ \\
\hline F14 & 0 & 1 & 0 & 95 & -1.68 & 1.16 & $155.0 \pm 1.8$ & $0.10 \pm 0.01$ & $-13.0 \pm 0.5$ & $90.84 \pm 1.35$ \\
\hline $\mathbf{F} 15^{\mathrm{a}}$ & 0 & 1 & 0 & 95 & 0 & 2.0 & $158.6 \pm 1.7$ & $0.08 \pm 0.00$ & $-13.6 \pm 0.5$ & $90.17 \pm 0.31$ \\
\hline F16 $^{\mathrm{a}}$ & 0 & 1 & 0 & 95 & 0 & 2.0 & $160.7 \pm 2.1$ & $0.10 \pm 0.02$ & $-13.9 \pm 0.7$ & $91.61 \pm 1.72$ \\
\hline
\end{tabular}

${ }^{\mathrm{a}}$ Centre points 


\section{Interactions Studies}

To assess the physical state of the PGZ and the possible interactions between the drug and the polymer, X-ray spectroscopy came into play, backed by FTIR spectral measurements and differential scanning calorimetry (DSC) analysis.

X-ray spectroscopy was used to analyse the state (amorphous or crystalline) of the PGZNSs. Powder of PGZ, PLGA-PEG, and NSs was sandwiched between films of polyester and exposed to $\mathrm{CuK}$ " radiation $(45 \mathrm{kV}, 40 \mathrm{~mA}, \lambda=1.5418 \AA$ ) in the range $2 \Theta / \Theta$ scands from $2^{\circ}$ to $60^{\circ} 2 \Theta$ with a step size of $0.026^{\circ} 2 \Theta$. The measuring time was 200 seconds per step.

To obtain FTIR spectra of PGZ, PLGA-PEG, and NSs a Thermo Scientific Nicolet iZ10 with an ATR diamond and DTGS detector were used. The scanning range was 525-4000 $\mathrm{cm}^{-1}$.

DSC analysis was performed using a DSC 823e System (Mettler-Toledo, Barcelona, Spain). A pan with indium (purity $\geq 99.95 \%$; Fluka, Switzerland) proved ideal to check the calibration of the calorimetric system. An empty pan served as a reference. The DSC measurements were carried out on the PGZ, PLGA-PEG and NSs. On heating the samples (2.32-2.95 $\mathrm{mg}$ ) from $25^{\circ} \mathrm{C}$ to $235^{\circ} \mathrm{C}$ at $10^{\circ} \mathrm{C} / \mathrm{min}$ in a nitrogen atmosphere it was possible to evaluate the data from the peak areas using the Mettler STARe V 9.01 DB software (Mettler-Toledo).

\section{Release Profile of PGZ-NSs}

The Franz diffusion cell technique enabled PGZ release studies from NSs to be carried out giving models that explained the release of the drug from the polymer matrix. Behind this there is the direct dispersion of the NSs in the dialysis medium complying with sink conditions (20). For 24 hours a dialysis membrane was hydrated and then placed in the 
Franz diffusion cell. The temperature of the medium and speed of the paddle were set at $32.0 \pm 0.5^{\circ} \mathrm{C}$ and $100 \mathrm{rpm}$, respectively. The comparisons of the PGZ-NSs formulations were made with free drug $(1 \mathrm{mg} / \mathrm{ml})$ dissolved in DMSO and a phosphate buffer solution (PBS) (60:40), receptor solution (RS) at $\mathrm{pH} 7.4$ for 15 hours to allow an estimated modeling. A volume of $0.3 \mathrm{ml}$ of formulations was put in the donor compartment and the same medium was introduced for the solubilization of free drug in the receptor compartment. A volume of $0.3 \mathrm{ml}$ was withdrawn from the receptor compartment at marked times and its place taken by an equivalent volume of RS at the same temperature. HPLC provided the released PGZ concentration. Values are reported as the average \pm SD using six replicates. At each point of time, the released PGZ content was evaluated and the data were adjusted to the most common kinetic models (21). In each case Akaike's information criterion (AIC) was determined: it was to be an indicator of the model's suitability for a given set of data (22).

\section{Corneal and Scleral Permeation Studies}

Ex vivo corneal and scleral permeation experiments were carried out with pigs (male, weight $30-40 \mathrm{~kg}$ and group $\mathrm{n}=6$ ). These were supervised by veterinary officials and were in accordance with the ethics committee of animal experimentation at the University of Barcelona. The pigs were anesthetized with intramuscular administration of ketamine $\mathrm{HCl}(3 \mathrm{mg} / \mathrm{kg})$, xylazine $(2.5 \mathrm{mg} / \mathrm{kg})$ and midazolam $(0.17 \mathrm{mg} / \mathrm{kg})$. Once sedated, the Propofol (3 mg/kg) was administered by auricular vein and immediately afterwards they were intubated and maintained under anesthesia inhaled with isoflurane. In order to induce pig euthanize, $250 \mathrm{mg} / \mathrm{Kg}$ of sodium pentobarbital were administered through the auricular ear vein under deep anesthesia. The cornea and sclera of the animals were cut away and placed in an artificial tear solution. The assay was carried out using Franz diffusion cells and the tissue was fixed between the donor and receptor compartment. The 
area in which the permeation could work was $0.64 \mathrm{~cm}^{2}$. The receptor compartment was filled with a freshly prepared transcutol/water solution $(60 / 40 \mathrm{v} / \mathrm{v})$ and kept at $32.0 \pm 0.5$ ${ }^{\circ} \mathrm{C}$ and stirred continuously for 6 hours. Volumes of $0.2 \mathrm{ml}$ from a solution containing 1 $\mathrm{mg} / \mathrm{ml}$ of PGZ-NSs and free drug solution (solubilized at the same receptor medium) were added to the donor compartment (covered with parafilm so that there should be no evaporation). A volume of $0.2 \mathrm{ml}$ was taken from the receptor compartment at predetermined times and in its place an equivalent volume of transcutol/water solution was introduced at the same temperature. The cumulative PGZ amount permeated per unit area $\left(\mu \mathrm{g} / \mathrm{cm}^{2}\right)$ was worked out at each time in point from the PGZ concentration in the receiving medium and a graph drawn up as a function of time (h). All experiments were carried out under sink conditions. Samples were analyzed by HPLC.

\section{PGZ Amount Retained in the Cornea}

PGZ quantification in the ocular tissues was realized after 6 hours of the experiment. The cornea and sclera were cleaned using a $0.05 \%$ solution of sodium lauryl sulfate and thoroughly rinsed with distilled water. They were weighed and during 30 minutes PGZ was extracted with methanol under sonication using an ultrasound bath. PGZ levels were expressed as $\mu \mathrm{g} /\left(\mathrm{g} \cdot \mathrm{cm}^{2}\right)$ of the cornea or sclera permeated and retained through the tissues. HPLC determined the PGZ. Results are reported as the average \pm SD for the PGZ amount permeated and retained on each tissue, respectively.

\section{Ocular permeation parameters}

At every marked time the cumulative drug amounts $\left(Q_{t}\right)$ permeated through the cornea and sclera per unit area were noted. The retained amount $\left(Q_{r e t}\right)$ was calculated working from the amount extracted and the recovery percentage - information already available. The amount extracted $\left(Q_{\text {ext }}\right)$ was calculated at the end of the experiment from the contact 
area vs tissue weight, after sonication. PGZ flux $\left(\mathrm{J}_{s s}\right)$ through the cornea and sclera from each formulation was calculated setting out on a graph the cumulative drug amount permeating the cornea and sclera over time. The slope of the linear portion of the curve was found by linear regression analysis and dividing it by the diffusion area. The permeability coefficient of the drug at the steady state $\left(K_{p s s}\right)$ from formulations was worked out using (Eq. 4):

$$
K_{p s S}=\frac{J_{S S}}{c_{o}}
$$

being $J_{s s}$ the flux and $c_{o}$ the initial drug concentration in the donor phase.

\section{Ocular Hydration Levels}

At the end of the corneal permeation assays, the level of ocular hydration (HL (\%)) of cornea assessed was determined. Then, each cornea was gently released from the sclera ring, washed, weighed and desiccated at a constant weight and dried at $80{ }^{\circ} \mathrm{C}$ and then reweighed. HL (\%) was arrived at in line with a previous study (9) using (Eq. 5):

$$
H L(\%)=\left[1-\left(w_{d} / w_{w}\right)\right] \cdot 100
$$

where $w_{d}$ is weight of the cornea after being dried and $w_{w}$ is the weight before being dried. Results are reported as the average \pm SD of six replicates.

\section{Studies of Ocular Tolerance in vitro and in vivo}

\section{HET CAM $^{\circledR}$}

When it came to evaluating the ocular tolerability of the developed formulation, the modified hen's egg chorioallantoic membrane (HET-CAM) test was carried out. The potential irritancy of compounds may be detected by observing adverse changes that occur in the CAM of the egg after exposure to test chemicals (23). Briefly, fertilized hens' 
eggs were maintained at a temperature of $12 \pm 1{ }^{\circ} \mathrm{C}$ not less than 24 hours before placing them in the incubator at a controlled temperature $\left(37.8^{\circ} \mathrm{C}\right)$ and humidity $(50-60 \%)$, to stay there over the days of incubation.

The shell was cut a little above the marked line of the chorioallantoic membrane (first this section of shell was removed). The inner membrane directly in contact with the CAM was dampened with $1 \mathrm{ml}$ of $0.9 \%$ saline solution, added with a pipette. The inner membrane was then removed with great care. It was important that no harm was done to the blood vessels, then it was possible to see the chorioallantoic membrane below. A volume of $0.3 \mathrm{ml}$ of the studied formulation was then added directly by pipette onto the CAM. Analysis, hemorrhaging and/or coagulation at different times over a 5 minute period after the application of the test solution were written down, and any effect that stood out was compared with the controls: saline (negative) and sodium hydroxide (positive) solutions. Each test was performed 6 times. Data were analyzed as the average $\pm \mathrm{SD}$ of the time at which the injury had happened ( $\mathrm{n}=6 /$ group). The scores were recorded according to the scoring schemes as described previously (7). (Table S2: a. Supplementary data).

\section{Draize Test}

Tests on pigs (male, weight 30-40 kg) revealed the extent of the irritancy of the PGZ-NSs formulations. This assay was the primary eye irritation test following the methods set down by Draize (1944) and Kay et al., (1962), (n=6/group) (24,25). A volume of $0.05 \mathrm{ml}$ of the sample from a concentration of $1 \mathrm{mg} / \mathrm{ml}$, was administered in the conjunctival sac of the right eye: the untreated contra-lateral eye was a control. The level of irritation was assessed 1 hour after application of the formulation. An analysis was carried out of ocular lesions in the cornea (opacity), iris (swelling, hemorrhage) and conjunctiva (inflammation, congestion, chemosis and discharge) guided by the ocular irritation index 
(OII) by visual assessment looking for any changes in the cornea, conjunctiva and iris. (Table S2: b. Supplementary data).

\section{In vivo Bioavailability Study}

In order to investigate ocular bioavailability and disposition of PGZ, 6 hours after topical administration of $0.05 \mathrm{ml}$ of PGZ-NSs from a concentration of $1 \mathrm{mg} / \mathrm{ml}$, the pigs were euthanized and the ocular tissues, including the retina, cornea, lens, sclera, choroid, iris, aqueous humor and vitreous humor were isolated from the eyes and kept at $-80{ }^{\circ} \mathrm{C}$ until liquid chromatography quantification of the PGZ amount - mass spectrometry (LC-MS) as the average $\pm \operatorname{SD}$ ( $\mathrm{n}=6$ /group).

\section{Cell Culture Cell Line (Y-79)}

The cytotoxicity of PGZ-NSs in comparison to the free PGZ was carried out in the Y-79 cell line, exposed to different concentrations which went from 2 to $100 \mu \mathrm{g} / \mathrm{ml}$, using the Alamar blue $(\mathrm{AB})$ assay. $\mathrm{AB}$ (resazurin) is a sensitive oxidation-reduction metabolic indicator. Once it has entered the cells and in the presence of metabolic reducing equivalent molecules (originating from cell metabolism), it changes its coloration from blue to rose (and thus the absorbance spectra shifts). The Y-79 cells were kept in RPMI1640, supplemented with $10 \%$ (v/v) fetal bovine serum (FBS), 2 mM L-glutamine, and antibiotics (100 U/ml penicillin and $100 \mu \mathrm{g} / \mathrm{ml}$ of streptomycin) in an atmosphere of $5 \%$ $\mathrm{CO}_{2}$ in air at $37^{\circ} \mathrm{C}$. The cells were centrifuged, re-suspended in culture media, the number recorded and seeded, once the appropriate dilution had been made, at $1 \cdot 10^{5}$ cells $/ \mathrm{ml}$ in poly-L-lysine pre-coated 96-well plates (100 $\mu \mathrm{g} /$ well) for adherence, which was achieved in about 24 hours. After adherence, the culture medium was taken away and test solutions added in. PGZ-NSs and free PGZ were diluted with FBS-free culture medium to achieve the desired final concentrations (test solutions), and then introduced to the cells $(0.1$ 
$\mathrm{ml} /$ well). Microplates were placed in the incubator, and the cells were in contact with the test solutions for 24 or 48 hours. Once the exposure time was over, the media containing the NSs and the control were taken away and replaced by FBS-free medium supplemented with $10 \%(\mathrm{v} / \mathrm{v})$ of $\mathrm{AB}$. The absorbance readings took place about 4-5 hours after $\mathrm{AB}$ addition, at $570 \mathrm{~nm}$ (reduced form) and $620 \mathrm{~nm}$ (oxidized form). A Multiskan EX microplate reader was employed (MTX Labsystems, USA). The cell viability was worked out by the percentage of $\mathrm{AB}$ reduction, using equations as recommended by $\mathrm{AB}$ manufacturers as described previously (26).

\section{In vivo Anti-inflammatory Assay}

To assess inflammation prevention, PGZ-NSs were tested in pigs (n=6/group). Firstly, there was just one instillation of a single dose administered at $0.05 \mathrm{ml}$ of PGZ-NSs (concentration of $1 \mathrm{mg} / \mathrm{ml}$ ) or $0.9 \%(\mathrm{w} / \mathrm{v})$ isotonic saline solution (control) in the conjunctival sac of the right eye. The contralateral eye acted as the untreated control. After 30 minutes, ocular inflammation was induced administering $0.05 \mathrm{ml}$ of SA $0.5 \%$ (w/v) dissolved in a phosphate buffer solution ( $\mathrm{pH} 7.4)$ instilled in the right eye. From the first reading after 30 minutes, thereafter every 30 minutes a new reading was made of the inflammation to measured it up to three hours (180 minutes) after the instillation of SA. The level of inflammation was quantified through ocular changes, in line with a modified Draize scoring system (27), which are shown as the sum of the inflammation scores expressed as the average \pm SD of six replicates.

\section{Statistical Analysis}

The GraphPad Prism 6.0 software package was used to analyze the data. The student's $t$ test was used for two-group comparisons and Statgraphics Plus 5.1 software. To analyze the factorial design, statistical evaluation of data was carried out using a one-way analysis 
of variance (ANOVA) test. Differences were taken as statistically significant when the $p$ value fell below 0.05 . All of the data are presented as the average \pm SD.

\section{RESULTS AND DISCUSSION}

\section{Factorial Design and Characterization of PGZ-NSs}

The experiments design was used to optimize the parameters, in order to obtain particles suitable for ocular administration. The results of factorial design ranged from $\mathrm{Z}_{\mathrm{av}} 148.9$ to $165.4 \mathrm{~nm}$ with monomodal distribution, PI values between 0.08 and 0.17 (Table I). There were no statistically significant effects noticeable when morphometric properties were indicated as responses. $\mathrm{ZP}$ values ranged from -10.1 to $-15.6 \mathrm{mV}$, and were greatly influenced by PGZ concentration (Fig. 1A), with a coefficient of determination of 0.93 . A low PGZ concentration allows the obtaining of high negative ZP values which can increase the stability of these systems. In the Pareto diagram (Fig. S1: B. Supplementary data) the influence of PGZ concentration on the ZP was observed. The EE values ranged from $81.19 \%$ to $94.47 \%$, and showed a significant decrease at high PGZ concentrations (Fig. 1B). This could be put down to the fact that the PGZ concentration indirectly influences encapsulation $\left(r^{2}=0.94\right)$. For these reasons, in order to obtain an equilibrium between stability and high EE, the replicate formulation (F15 and F16) was selected (1 $\mathrm{mg} / \mathrm{ml}$ of PGZ, $95 \mathrm{mg}$ of polymer, $2 \%$ of Tw 80 and $\mathrm{pH}$ of 4.5). 
(A)

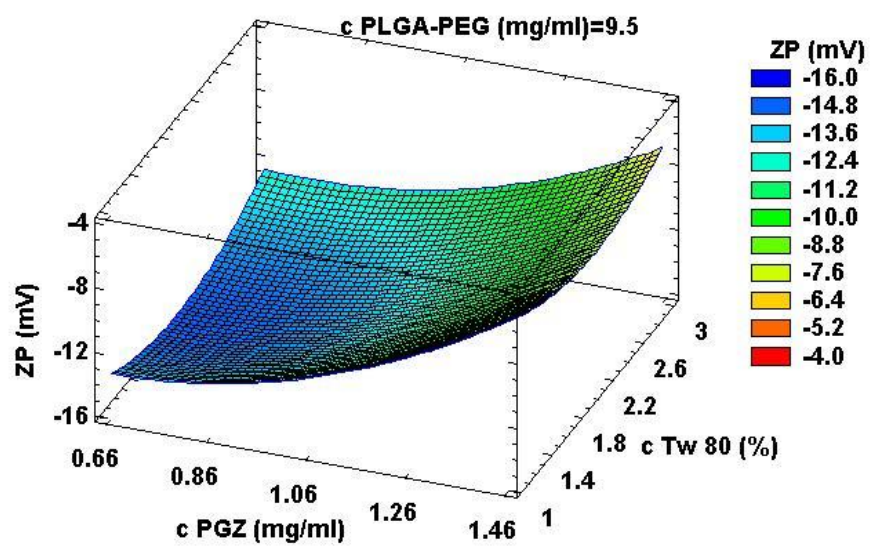

(B)

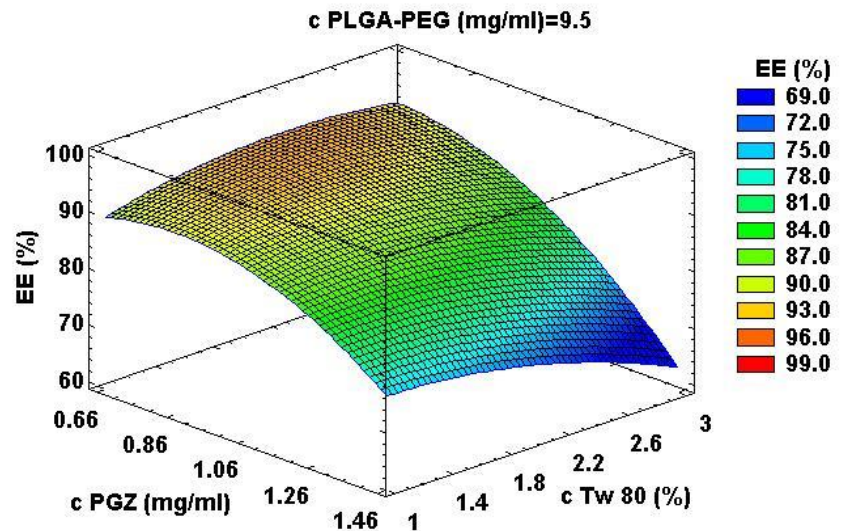

Fig.1.:

The optimized PGZ loaded PLGA-PEG NSs, made by solvent displacement technique, showed a $\mathrm{Z}_{\mathrm{av}}$ around $160.0 \pm 1.3 \mathrm{~nm}$, with PI values in the range of monodisperse systems (PI < 0.1) and high association efficiency (92\%), which is suitable for ophthalmic application. The $\mathrm{ZP}$ that could be considered as a parameter of stability and mucoadhesion of these systems was $-13.9 \mathrm{mV}$, indicating an adequate short-time stability. To determine the size and surface morphology of the optimized PGZ-NSs TEM was the method chosen (Fig. 2), evidencing that PGZ-NSs are spherical in morphology. 


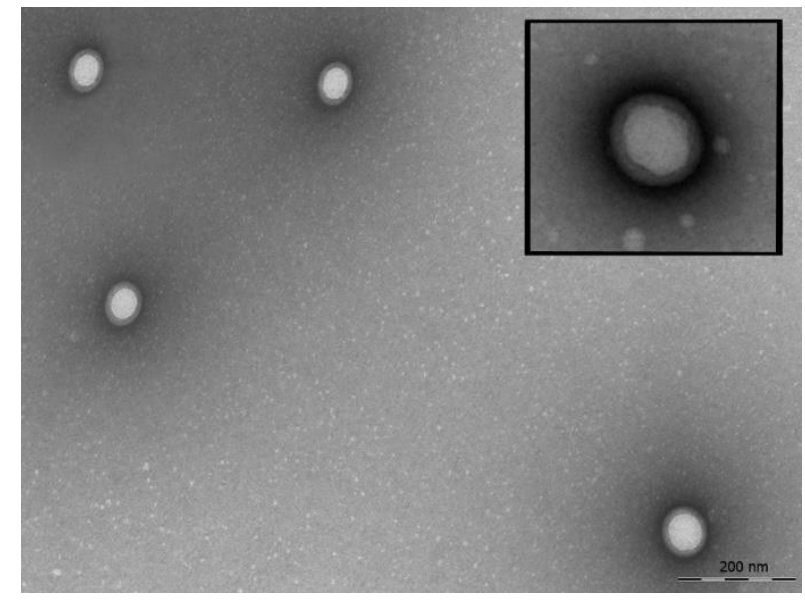

Fig. 2.:

\section{Interactions Studies}

The in vivo and in vitro release of drugs from delivery systems could be influenced by the physical state of drugs in the colloidal systems. Therefore, different combinations of drug/polymer could coexist in the bulk, and these would be: (i) amorphous drug in either an amorphous or a semi-crystalline polymer or (ii) crystalline drug in either an amorphous or a semi-crystalline polymer. This leads on to it being possible that the drug may be present as either a solid solution or as a solid dispersion in an amorphous or semicrystalline polymer (19). Herein lies why it is necessary to be aware of the interactions between the polymer and the drug.

Fig. 3A sets out the X-ray diagrams of PGZ, PLGA-PEG and NSs. The X-ray spectrum of PGZ powder showed sharp crystalline peaks, whereas PLGA-PEG and NSs, according to their profiles, were amorphous. These results suggested that when the drug was loaded under the form of NSs, showed a similar polymer profile. This fact can be clearly seen in the detail of Fig. 3A.

FTIR analysis suggests that there is no evidence of new covalent bonds, between the drug and the polymer (Fig. S2. Supplementary data). These results are what can be expected in line with those set forth by other authors (21). 
The DSC profiles of PGZ showed an endothermic event between $153.63^{\circ} \mathrm{C}$ to $209.15^{\circ} \mathrm{C}$, which corresponded to solid-liquid transition ( $\mathrm{T}_{\max }$ of $196.54^{\circ} \mathrm{C}$ and $\Delta \mathrm{H}$ of $138.00 \mathrm{~J} / \mathrm{g}$ ), followed by another thermic event that corresponded to drug decomposition. The polymer showed an endothermic event attributed to glass transition $\left(\mathrm{T}_{\mathrm{g}}\right)$ (midpoint ISO of 43.81 ${ }^{\circ} \mathrm{C}$ and onset of $43.03^{\circ} \mathrm{C}$ ). PGZ-NSs showed a thermic event that corresponds to glass transition $\left(\mathrm{T}_{\mathrm{g}}\right)$ of the polymer in the form of NSs (midpoint ISO of $40.98{ }^{\circ} \mathrm{C}$ and onset of $38.82{ }^{\circ} \mathrm{C}$ ). The $\mathrm{T}_{\mathrm{g}}$ parameters decrease in the NSs is probably due to a plastic effect exerted by the drug on the polymer. As there is no endotherm corresponding to the drug fusion this indicates that the PGZ is in the form of either a molecular dispersion or a solid solution (Fig. 3B). These results are similar to those obtained by lipophilic drugs in the form of NSs (19).

(A)

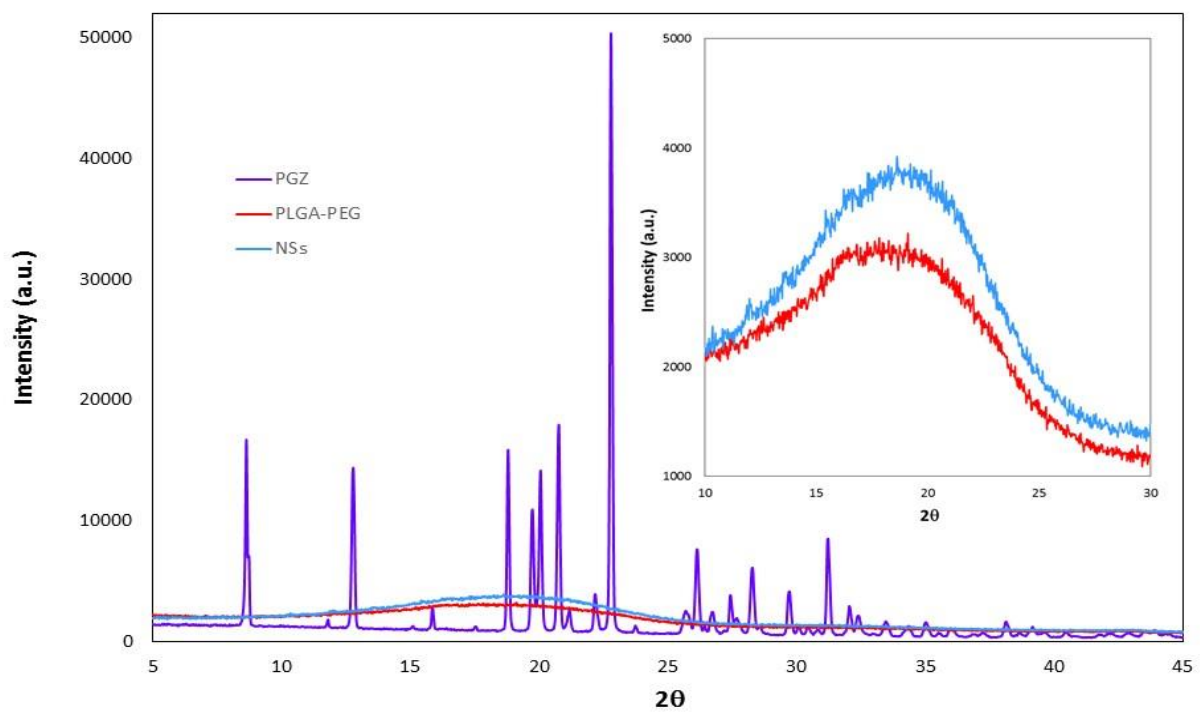

(B) 


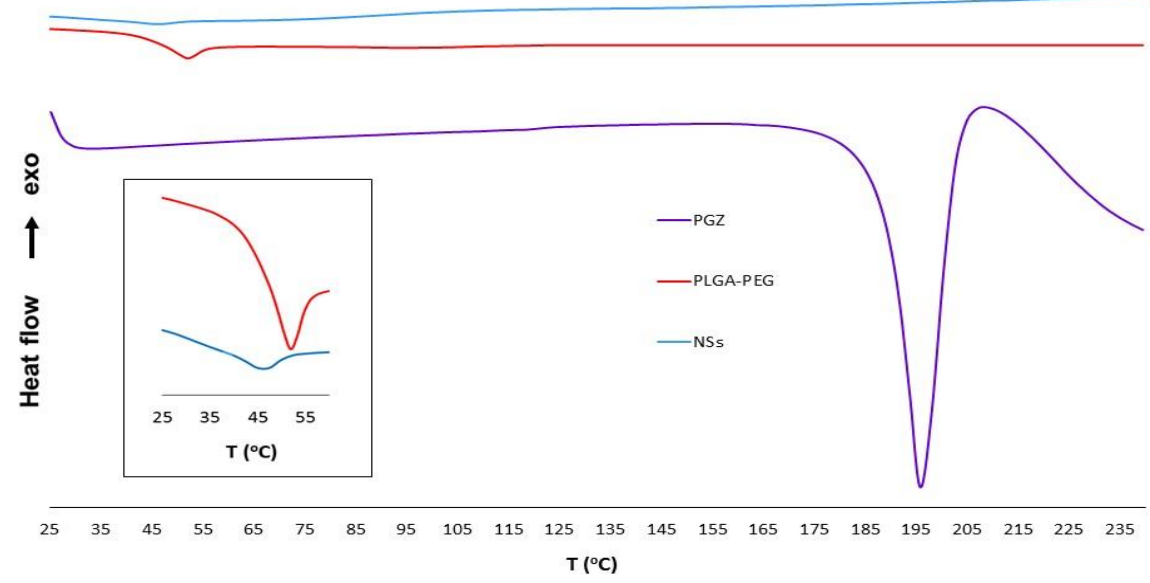

Fig. 3.:

\section{In vitro Release Profile}

The release profiles of PGZ-NSs and free PGZ (Fig. 4A), performed at $32{ }^{\circ} \mathrm{C}$, were the same model for both formulations. However, free drug was released faster than PGZ-NSs. In the first hour, both formulations showed fast release profiles, with $87.35 \mu \mathrm{g}$ to free solution and $57.80 \mu \mathrm{g}$ to PGZ-NSs, there being enough quantity to permeate the ocular tissues. This result contrasts with the results of permeations. Since the NSs adhere to the cornea and sclera, and due to the fact that this also happens on the surface of tissues, there is more contact and penetration of the drug $(10,28)$.

The maximum amounts released $\left(Y_{\max }\right)$ from the drug free solution and PGZ-NSs were 221.70 and $229.60 \mu \mathrm{g}$ respectively, being significantly different among the formulations ( $p$ value $=0.0487)$. This assay showed a profile Fick's passive diffusion with a constant of dissolution (K) of $0.44 \mathrm{~h}^{-1}$ and $0.28 \mathrm{~h}^{-1}$ for free drug and NSs, respectively. The free solution has a release rate 1.6 times faster than NSs, this being significantly slower $(p=$ 0.0001 ), although at 30 minutes the PGZ released was 38.83 versus $55.23 \mu \mathrm{g}$ from free solution. This leads to the conclusion that PGZ release from NSs follows the same course 
as a concentration gradient pattern, based on the Fick's first law. These results are in line with other studies (8).

In general, the release of the drug from PLGA-PEG particles can occur through diffusion, erosion of the polymer or a combination thereof. If the diffusion of the drug progresses at a higher speed than the degradation of the matrix, the mechanism of drug release makes itself felt mainly by diffusion (29).

After 11 hours, a gradual release behavior was shown, where the entrapped PGZ very gradually diffused out of the polymeric matrix into the release medium. According to the AIC and coefficient of determination $\left(\mathrm{r}^{2}\right)$ values obtained, the best fitting for all formulations was the one phase exponential association (Table II). 
Table II. Parameters of release profile

\begin{tabular}{lcccc}
\hline \multicolumn{1}{c}{ Kinetics Models } & \multicolumn{2}{c}{ Free PGZ } & \multicolumn{2}{c}{ PGZ-NSs } \\
\cline { 2 - 5 } & AIC & $\mathbf{r}^{\mathbf{2}}$ & AIC & $\mathbf{r}^{\mathbf{2}}$ \\
\hline $\begin{array}{l}\text { One exponential association } \\
Q_{t}=Q_{\infty} \cdot\left(1-e^{-K \cdot t}\right)\end{array}$ & 62.3 & 0.974 & 111.3 & 0.977 \\
$\begin{array}{l}\text { Hyperbola } \\
Q_{t}=Q_{\infty} \cdot t /(K+t)\end{array}$ & 63.0 & 0.971 & 113.2 & 0.974 \\
$\begin{array}{l}\text { Zero order } \\
Q_{t}=K_{0} \cdot t+Q_{\infty}\end{array}$ & & & \\
Korsmeyer Peppas & 79.0 & 0.713 & 138.5 & 0.814 \\
$Q_{t}=K \cdot t^{n}$ & & & \\
& & & \\
$Q_{t}=$ cumulative amount of drug release at time $t ; Q_{\infty}=$ maximum amount of drug released; $K_{0}, \mathrm{~K}=$ release rate constants; $\mathrm{t}=$ time \\
in hours; $n$ is the diffusion release exponent; $\mathrm{r}^{2}=$ determination coefficient; AIC $=$ Akaike's information criterion.
\end{tabular}




\section{Ex vivo Permeation Studies}

The transcorneal and transcleral permeation studies (Fig. 4B and C, respectively), carried out for 6 hours, showed different profiles for each one. In the statistical analysis of $J_{s s}$ and $Q_{r e t}$, cornea and scleral expressed significant differences $(p<0.0001)$ when one compares the PGZ-NSs versus free PGZ (Table III). The value of $J_{s s}$ of the PGZ in cornea and sclera were similar for free drug. The PGZ from NSs permeates less through the cornea than sclera, showing that the cornea has a barrier effect three times higher than the sclera (30). This is a consequence of the cornea having a regular cellular order, while the cells of the sclera have a more irregular cellular arrangement (31). Another factor is the physicochemical properties of the molecule, which better interacts with scleral tissue, as well as other molecule properties favoring good scleral permeability, such as Triamcinolone (32). The permeability from NSs is important because these systems facilitate the permeation of faintly water-soluble drugs, in this case the PGZ.

The $\mathrm{K}_{p s s}$ is proportional to the $J_{s s}$, therefore this parameter is smaller in the corneal than than in scleral tissue. To quantify both tissues, $Q_{r e t}$ of PGZ from NSs was higher than free drug. In both the $Q_{\text {ret }}$ of PGZ were similar in the corneal tissue.

The $\mathrm{Q}_{\text {ret }}$ in the sclera showed significant differences from PGZ-NSs to free drug 87.9 and $72.47 \mu \mathrm{g} /\left(\mathrm{g} \cdot \mathrm{cm}^{2}\right)$, respectively. The high permeability and accumulation of PGZ from NSs in the scleral tissue could be attribute to a better activity of these systems for posterior ocular diseases, as well as uveitis, probably due to high sclera permeability (33). These findings are in line with those obtained previously, which revealed that the PGZ could be effective for the treatment to endogenous uveitis (3). In another study, it was shown that PPAR $\alpha$ agonist medical care significantly lessens inflammatory cell infiltration, total protein concentration, vessel density, and the development of inflammatory cytokine 
(34). After 1 hour, the permeated quantify of PGZ from NSs in sclera was $5.6 \mu \mathrm{g}$ in comparison with $1 \mu \mathrm{g}$ in the cornea, it being sufficient to cross both tissues.

(A)

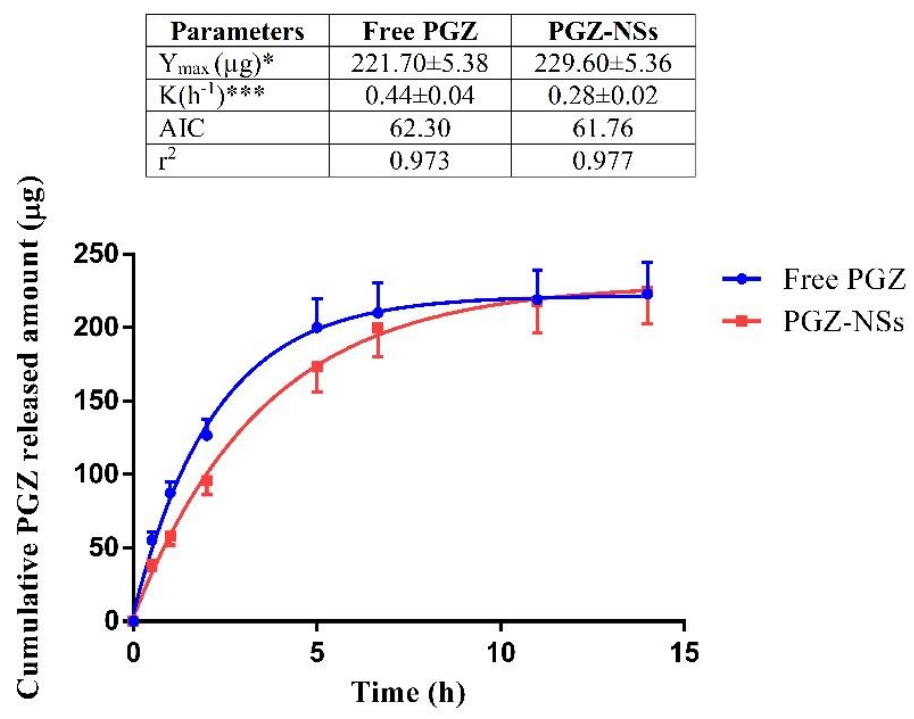

(B)

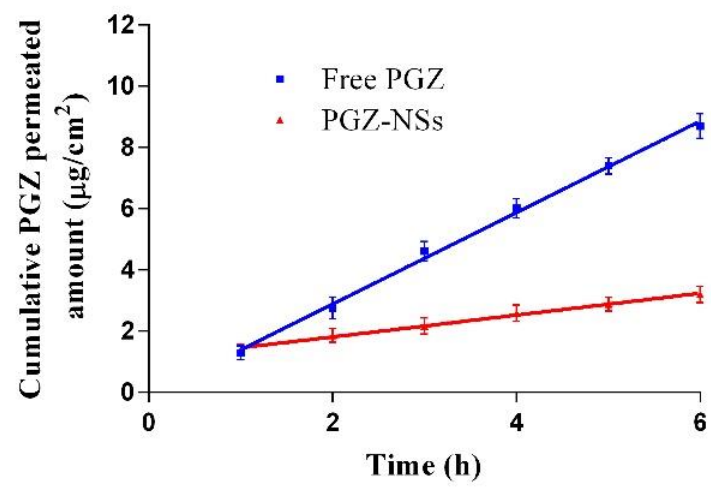


(C)

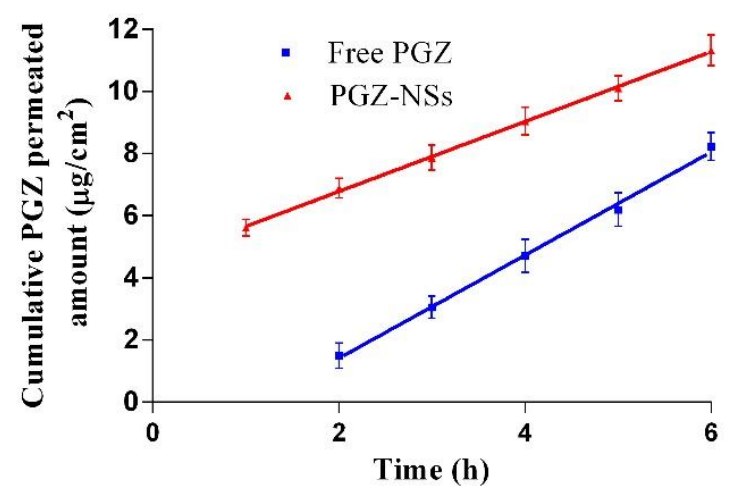

Fig. 4.: 
Table III. Parameters of ocular permeation.

\begin{tabular}{lllcc}
\hline & \multicolumn{2}{c}{ Corneal permeation } & \multicolumn{2}{c}{ Scleral permeation } \\
\cline { 2 - 5 } & Free PGZ & PGZ-NSs & Free PGZ & PGZ-NSs \\
\hline$J_{s s}\left(\mu \mathrm{g} /\left(\mathrm{cm}^{2} \cdot \mathrm{h}\right)\right)$ & $2.33 \pm 0.04^{\mathrm{a}}$ & $0.55 \pm 0.01$ & $2.59 \pm 0.05^{\mathrm{b}}$ & $1.75 \pm 0.01$ \\
$K_{p}(\mathrm{~cm} / \mathrm{h})$ & $2.33 \pm 0.02$ & $0.56 \pm 0.03$ & $2.59 \pm 0.03$ & $1.75 \pm 0.01$ \\
$Q_{\text {ext }}\left(\mu \mathrm{g} /\left(\mathrm{g} \cdot \mathrm{cm}^{2}\right)\right)$ & $22.63 \pm 1.34$ & $23.08 \pm 2.14$ & $16.68 \pm 2.43$ & $20.23 \pm 3.05$ \\
$Q_{\text {ret }}\left(\mu \mathrm{g} /\left(\mathrm{g} \cdot \mathrm{cm}^{2}\right)\right)$ & $26.64 \pm 0.07^{\mathrm{a}}$ & $27.17 \pm 0.05$ & $72.47 \pm 0.12^{\mathrm{b}}$ & $87.90 \pm 0.25$
\end{tabular}

$J_{s S}=$ Flux; $K_{p}=$ permeability coefficient of the drug at the steady state; $Q_{\text {ext }}=$ extracted amount; $Q_{\text {ret }}=$ retained amount. Parameters values are expressed as average $\pm \mathrm{SD} ;{ }^{\mathrm{a}, \mathrm{b}} p<0.0001$. $^{\mathrm{a}}=$ Comparison between Free PGZ vs PGZ NSs with cornea tissue. ${ }^{b}=$ Comparison between Free PGZ vs PGZ NSs with sclera tissue. 


\section{Ocular Hydration Levels}

The HL (\%) is frequently taken as a parameter to measure the harm done to the cornea tissue. The normal cornea has a hydration level of 76-80\% (35). A HL level of 3-7 \% greater than the normal value indicates damage to the epithelium or endothelium. The HL percentage obtained for PGZ-NSs $(79.82 \%)$ has shown that it can be accepted as being within an adequate range, and does not cause any harm to the corneal tissue (Table S1: Supplementary data). This result is in accordance with that obtained for controlled release systems and transcutol ${ }^{\circledR} \mathrm{P}(7)$.

\section{Tolerance Ocular Assays (HET CAM ${ }^{\circledR}$ and Draize Test)}

The in vitro potential irritation was detected by the HET CAM test. The CAM is a membrane with such vascularity that it has structure similar to other highly vascularized tissues. Moreover, the conjunctiva is an ideal model for ocular irritation studies. A volume of $0.3 \mathrm{ml}$ of PGZ-NSs was administered to the CAM for 5 minutes and there was no irritation. Hemorrhage, vascular lysis or coagulation were not seen in any way. An OII of 0.4 was the score, indicating that these systems are safe for application to the eye.

In the in vivo studies pigs were used in order to analyze the ocular tolerance level (Draize test) of the PGZ-NSs. No irritations or damaging effects were detected after the PGZ-NSs had been instilled, being the OII $=0$ at all the points. This assay indicated an optima ocular tolerance and was reinforced by the results obtained from the Hen's Egg test (HETCAM). In accordance with other authors, polymeric NSs are safe for ocular administration, because of their biocompatible material $(7,10,21,36)$ (Table S3: Supplementary data). 


\section{In vivo Bioavailability Study}

In order to determine the in vivo bioavailability studies, the PGZ-NSs were administered post topically for 6 hours, quantified in different eye tissues. Different levels of PGZ were obtained from the PGZ-NSs in the ocular tissues as well as in the retina $(30.78 \mu \mathrm{g} / \mathrm{g})$, cornea $(20.52 \mu \mathrm{g} / \mathrm{g})$, lens $(13.45 \mu \mathrm{g} / \mathrm{g})$, sclera $(64.45 \mu \mathrm{g} / \mathrm{g})$, choroid $(159.67 \mu \mathrm{g} / \mathrm{g})$, iris $(117.25 \mu \mathrm{g} / \mathrm{g})$, aqueous humor $(9.60 \mu \mathrm{g} / \mathrm{ml})$ and vitreous humor $(9.20 \mu \mathrm{g} / \mathrm{ml})$. The values are expressed by mean \pm SD (n=6) (Fig. S4. Supplementary data).

These results are in accordance with those obtained in ex vivo corneal and scleral permeation studies. The PGZ exhibited higher levels in the scleral tissue, demonstrating that this drug could be useful to treat diseases affecting the posterior segment of the eye. However, through tests with small amounts of the drug it has been revealed that PGZ encapsulated in NSs achieves a release reaching many parts of the eye, including the retina. Further studies could be carried out to show new routes for the effectiveness of the agonist of PPAR $\gamma$ against ocular inflammatory diseases. The NSs could be a new strategy for the delivery of PGZ into the eye, also leading to a reduction in the adverse effects.

\section{Toxicity Assay}

The cell viability (expressed as \% of control) was carried out in human retinoblastoma cell line, Y-79, which were exposed to test the solution of PGZ-NSs, free PGZ and Tw80 2\%, diluted in FBS-free culture media to the final concentrations of 2, 10, 20, 50 and 100 $\mu \mathrm{g} / \mathrm{ml}$. The $\mathrm{AB}$ indicator was used to measure quantitatively the viability and propagation of the cells, rendering measurable the toxicity of tested agents/drugs in relation to nonexposed cells (control). It is possible to observe that the free drug did not produce any toxicity in the concentrations and exposure times analyzed, as the viability is always greater than $80 \%$ of control (Fig. 5). The PGZ-NSs, up to $10 \mu \mathrm{g} / \mathrm{ml}$, shows no toxicity, as 
cell viability is above $80 \%$, but doubling the concentration to $20 \mu \mathrm{g} / \mathrm{ml}$ shows a drastic reduction in cell viability with the percentage values being close to zero. Meanwhile, when the effect of Tw 80 (at $2 \%$ in the particles) was analyzed, it showed a similar profile of toxicity as that seen in PGZ-NSs up to $10 \mu \mathrm{g} / \mathrm{ml}$, with $80 \%$ of viability. The decrease of the level of cell viability (\%) induced by PGZ-NSs leads one to believe in an interaction of Tw 80 (not fully incorporated into the NSs or that leaked from the NSs) with the cell membranes, leading to their lysis. It is worth mentioning that this is an in vitro assay in which the living cells are directly exposed to the NSs and their components. The administering of these solutions directly to cells, and the systems used, exposing the cells' membranes to their components, is quite different from the administering of these solutions, with these systems, to the ocular organ. The application of these systems by the ocular route is non-toxic since the NSs are placed on the cornea and not directly on the cells (with the exposed cell membranes). According to previous reports, Tw 80, in comparison with other surfactants, showed the lowest cytotoxicity when tested in normal human fibroblast cultures (37).

These in vitro results contrast with the in vivo assays of ocular tolerance that show that NSs is non-irritant. This comparison corroborates the fact that the cornea works as a barrier, protecting the cells from NSs aggressive components while permitting the permeation of desired drugs. 


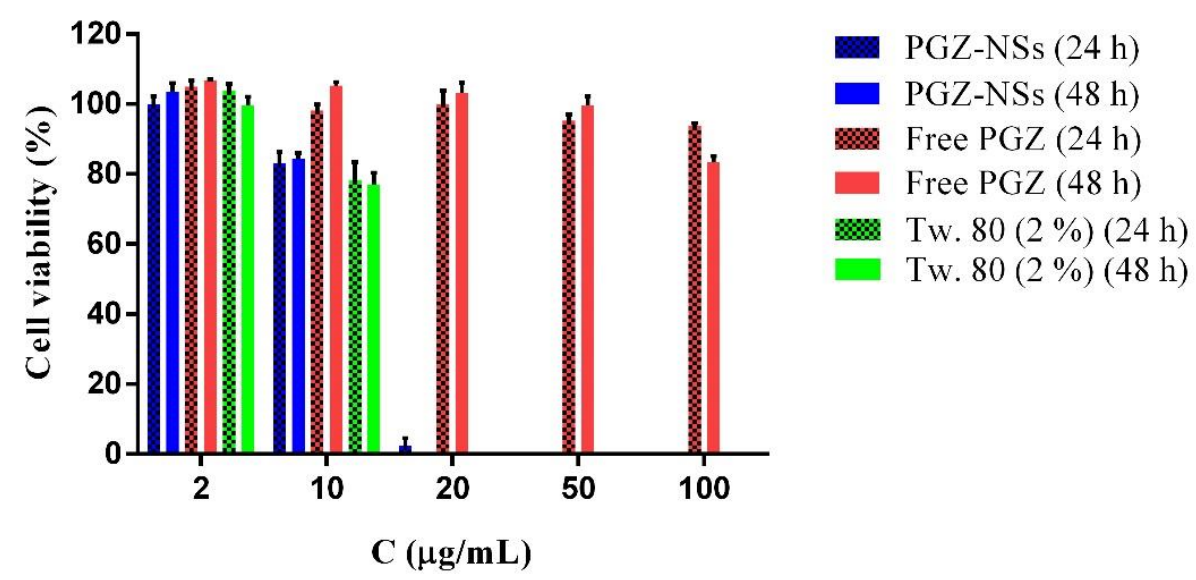

Fig. 5.:

\section{In vivo Anti-inflammatory Efficacy}

With the aim of evaluating the anti-inflammatory efficacy of PGZ-NSs in the prevention of ocular inflammation processes, PGZ-NSs were administered to the pigs 30 minutes before inducing inflammation with SA. PGZ-NSs showed significant differences regarding the positive control for the first 30 minutes after SA administration $(p=$ 0.0008), reducing the degree of conjunctival inflammation and iris hyperemia significantly, as shown in (Fig. 6). It may be due mainly to the quantity of PGZ provided by NSs in different tissues, resulting in a reservoir effect of the drug and promoting the continuity of the pharmacological action. These results demonstrate that PGZ in reduced doses is an effective therapeutic agent for ocular inflammation, possibly increasing drug effect by encapsulation in polymeric NSs.

It was showed by ocular permeation that free PGZ is distributed and retained in cornea and sclera in smaller proportions whereas PGZ-NSs provide higher drug levels in the sclera and cornea, as well as higher drug penetration in different tissues of the eye. A study showed that PGZ might improve impaired insulin signaling in the diabetic rat retina (38). Another study provide evidence that TZDs may have the potential to inhibit the progression of diabetic retinopathy (39). However, it has been described that an ophthalmic solution containing $0.1 \%$ PGZ inhibited inflammation, decreased the fibrotic 
reaction, and prevented corneal neovascularization in the cornea from the early phase after alkali burn injury in rats (4). However, in another study, it was shown that the PGZ inhibited intraocular concentrations of TNF- $\alpha$ and IL-6 in the endogenous uveitis model (2). These results demonstrated that PPAR $\gamma$ agonists may represent a way of moving forward with a treatment strategy focused on clinical applications in inflammatory processes and better wound healing. This data obtained could also suggest that the dispersion of the drug within the polymer in the form of NSs favors and thus adds to its ocular bioavailability.

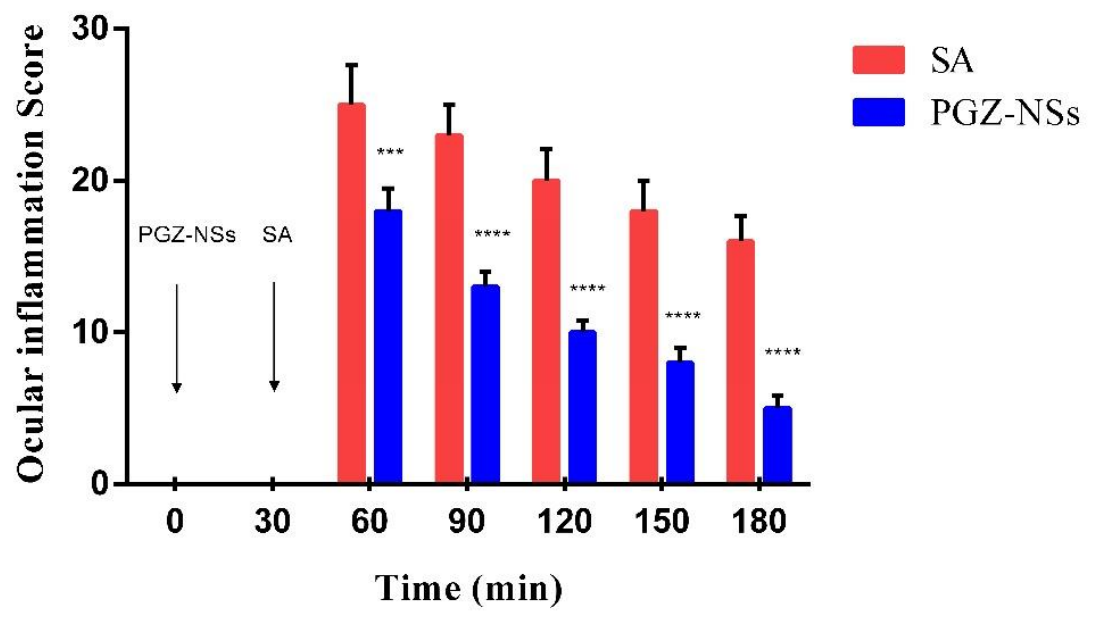

Fig. 6.:

\section{CONCLUSIONS}

In summary, our study demonstrated that PGZ-NSs developed by the displacement technique were characterized with an $\mathrm{Z}_{\mathrm{av}}$ appropriate for ocular administration (around $160 \mathrm{~nm})$, suitable ZP, high EE (92\%) and a good homogenization characteristic of monodisperse systems. The interaction studies showed that the potential of PGZ-NSs as a drug carrier system in which drug dispersion in the polymer increases the PGZ solubility. This is because the drug has a plastic effect on the polymer.

The release profile corresponds to Fick's passive diffusion, followed by a slower and continuous release. The permeation studies of NSs through the cornea and sclera 
demonstrated higher $Q_{r e t}$ of PGZ in the sclera $87.9 \mu \mathrm{g} /\left(\mathrm{g} \cdot \mathrm{cm}^{2}\right)$, which was corroborated in the bioavailability studies. The percentage of HL for the PGZ-NSs was within the normality parameters $(79.82 \%)$. No irritation or damaging effects were detected in the HET CAM and Draize Test. Cytotoxicity studies of the colloidal systems showed no toxicity up to $10 \mu \mathrm{g} / \mathrm{ml}$. The in vivo assay showed promising effects of the PGZ-NSs with respect to preventing inflammation. Taken together, the results of this study suggest that the PGZ-NSs show anti-inflammatory activity, which could be useful for the prevention of ocular inflammatory processes.

\section{ACKNOWLEDGMENTS}

This work has benefited from the backing of the Spanish Ministry of Science and Innovation (MAT2014-59134R). The authors would like to acknowledge Lidia Gómez Segura for her help with the in vivo study. Ms. Marcelle Silva de Abreu acknowledges the positive contribution of the Coordination for the Improvement of Higher Education Personnel (CAPES) - Brazil for the PhD scholarship.

\section{REFERENCES}

1. Pan H, Chen J, Xu J, Chen M, Ma R. Antifibrotic effect by activation of peroxisome proliferator- activated receptor $-\gamma$ in corneal fibroblasts. Mol. Vis. 2009;15:2279-86.

2. Yamamoto A, Kakuta H, Miyachi H, Sugimoto Y. Involvement of the retinoid X receptor ligand in the anti-inflammatory effect induced by peroxisome proliferatoractivated receptor agonist in vivo. PPAR Res. 2011;1-8.

3. Okunuki Y, Usui Y, Nakagawa H, Tajima K, Matsuda R, Ueda S, Hattori T, Kezuka T, Goto H. Peroxisome proliferator-activated receptor- $\gamma$ agonist pioglitazone suppresses experimental autoimmune uveitis. Exp Eye Res. 2013;116:291-297.

4. Uchiyama M, Shimizu A, Masuda Y, Nagasaka S, Fukuda Y, Takahashi H. An ophthalmic solution of a peroxisome proliferator-activated receptor gamma agonist prevents corneal inflammation in a rat alkali burn model. Mol Vis. 2013;19:213550 .

5. Calvo P, Vila-Jato JL, Alonso MJ. Evaluation of cationic polymer-coated 
nanocapsules as ocular drug carriers. Int J Pharm. 1997;153(1):41-50.

6. Araújo J, Vega E, Lopes C, Egea MA, Garcia ML, Souto EB. Effect of polymer viscosity on physicochemical properties and ocular tolerance of FB-loaded PLGA nanospheres. Colloids Surfaces B Biointerfaces. 2009;72(1):48-56.

7. Alvarado HL, Abrego G, Garduño-Ramirez ML, Clares B, Calpena AC, García ML. Design and optimization of oleanolic/ursolic acid-loaded nanoplatforms for ocular anti-inflammatory applications. Nanomedicine Nanotechnology, Biol Med. 2015;11(3):521-30.

8. Parra A, Mallandrich M, Clares B, Egea MA, Espina M, Garcia ML, Calpena AC. Design and elaboration of freeze-dried PLGA nanoparticles for the transcorneal permeation of carprofen: Ocular anti-inflammatory applications. Colloids Surfaces B Biointerfaces. 2015;136: 935-43.

9. Fangueiro JF, Calpena AC, Clares B, Andreani T, Egea MA, Veiga FJ, García ML, Silva AM, Souto EB. Biopharmaceutical evaluation of epigallocatechin gallateloaded cationic lipid nanoparticles (EGCG-LNs): In vivo, in vitro and ex vivo studies. Int J Pharm. 2016;502(1-2):161-9.

10. Ramos Yacasi GR, Garcia Lopez ML, Espina Garcia M, Parra Coca A, Calpena Campmany AC. Influence of freeze-drying and $\gamma$-irradiation in preclinical studies of flurbiprofen polymeric nanoparticles for ocular delivery using d-(+)-trehalose and polyethylene glycol. Int J Nanomedicine. 2016;11:4093-106.

11. De Campos AM, Sánchez A, Gref R, Calvo P, Alonso MJ. The effect of a PEG versus a chitosan coating on the interaction of drug colloidal carriers with the ocular mucosa. Eur J Pharm Sci. 2003;20(1):73-81.

12. Danhier F, Ansorena E, Silva JM, Coco R, Le Breton A, Préat V. PLGA-based nanoparticles: An overview of biomedical applications. J Control Release. 2012;161(2):505-22.

13. Cañadas C, Alvarado H, Calpena AC, Silva AM, Souto EB, Garcia ML, Abrego G. In vitro, ex vivo and in vivo characterization of PLGA nanoparticles loading pranoprofen for ocular administration. Int J Pharm. 2016;511(2):719-27.

14. $\mathrm{Xu} \mathrm{Y,} \mathrm{Du} \mathrm{Y.} \mathrm{Effect} \mathrm{of} \mathrm{molecular} \mathrm{structure} \mathrm{of} \mathrm{chitosan} \mathrm{on} \mathrm{protein} \mathrm{delivery}$ properties of chitosan nanoparticles. Int J Pharm. 2003;250(1):215-26.

15. Sánchez-López E, Egea MA, Cano A, Espina M, Calpena AC, Ettcheto M, Camins A, Souto EB, Silva AM, Garcia ML. PEGylated PLGA nanospheres optimized by design of experiments for ocular administration of dexibuprofen-in vitro, ex vivo and in vivo characterization. Colloids Surfaces B Biointerfaces. 2016;145:241-50.

16. Fessi H, Puisieux F, Devissaguet JP, Ammoury N, Benita S. Nanocapsule formation by interfacial polymer deposition following solvent displacement. Int $J$ Pharm. 1989;55(1):R1-4.

17. Satheeshkumar N, Shantikumar S, Srinivas R. 2014. Pioglitazone: A review of analytical methods. J Pharm Anal. 2014;4(5):295-302.

18. Clogston JD, Patri AK. Zeta Potential Measurement. In: McNeil SE, editor. Characterization of Nanoparticles Intended for Drug Delivery. Totowa, NJ: Humana Press; 2011;63-70.

19. Vega E, Gamisans F, García ML, Chauvet A, Lacoulonche F, Egea MA. PLGA Nanospheres for the Ocular Delivery of Flurbiprofen: Drug Release and Interactions. J Pharm Sci. 2008;97:5306-17.

20. Abrego G, Alvarado H, Souto EB, Guevara B, Bellowa LH, Parra A, Calpena A, García ML. Biopharmaceutical profile of pranoprofen-loaded PLGA nanoparticles containing hydrogels for ocular administration. Eur J Pharm Biopharm. 2015;95:261-70. 
21. Abrego G, Alvarado HL, Egea MA, Gonzalez-Mira E, Calpena AC, Garcia ML. Design of nanosuspensions and freeze-dried PLGA nanoparticles as a novel approach for ophthalmic delivery of pranoprofen. J Pharm Sci. 2014;103(10): 3153-64.

22. Yamaoka K, Nakagawa T, Uno T. 1978. Application of Akaike's information Criterion (AIC) in the evaluation of linear pharmacokinetic equations. $J$ Pharmacokinet Biopharm. 1978;6:165-75.

23. Luepke NP. Hen's egg chorioallantoic membrane test for irritation potential. Food Chem Toxicol. 1985;23(2):287-91.

24. Draize J, Woodard G, Calvery H. 1944. Methods for the study of irritation and toxicity of substances applied topically to the skin and mucous membranes. $J$ Pharmacol Exp Ther. 1944;82(3):377-90.

25. Kay JH, Calandra JC. Interpretation of eye irritation tests. J Soc Cosmet Chem. 1962;13:281-9.

26. Andreani $\mathrm{T}$ et al. Surface engineering of silica nanoparticles for oral insulin delivery: Characterization and cell toxicity studies. Colloids Surfaces B Biointerfaces. 2014;123:916-923.

27. Vega E, Antònia Egea M, Calpena AC, Espina M, Luisa García M. Role of hydroxypropyl-B-cyclodextrin on freeze-dried and gamma-irradiated PLGA and PLGA-PEG diblock copolymer nanospheres for ophthalmic flurbiprofen delivery. Int J Nanomedicine. 2012;7:1357-71.

28. Prausnitz MR, Noonan JS. Permeability of cornea, sclera, and conjunctiva: a literature analysis for drug delivery to the eye. J Pharm Sci. 1998;87(12):14791488.

29. Niwa T, Takeuchi H, Hino $T$, Kunou N, Kawashima Y. Preparations of biodegradable nanospheres of water-soluble and insoluble drugs with D,Llactide/glycolide copolymer by a novel spontaneous emulsification solvent diffusion method, and the drug release behavior. J Control Release. 1993;25:8998 .

30. Resende AP, Silva B, Braz BS, Nunes T, Gonçalves L, Delgado E. Ex vivo permeation of erythropoietin through porcine conjunctiva, cornea, and sclera. Drug Delivery and Translational Research. 2017;1-7.

31. Nguyen TD. Biomechanics of the Cornea and Sclera. In: Kassab GS, Sacks MS, editors. Structure-Based Mechanics of Tissues and Organs [Internet]. Boston, MA: Springer US; 2016;285-315.

32. Araújo J, Garcia ML, Mallandrich M, Souto EB, Calpena AC. Release profile and transscleral permeation of triamcinolone acetonide loaded nanostructured lipid carriers (TA-NLC): In vitro and ex vivo studies. Nanomedicine Nanotechnology, Biol Med. 2012;8(6):1034-41.

33. Wen HE, Hao J, Li SK. Characterization of Human Sclera Barrier Properties for Transscleral Delivery of Bevacizumab and Ranibizumab. J. Pharm Sci. 2013;102(3):892-903.

34. Shen W, Gao Y, Lu B, Zhang Q, Hu Y, Chen Y. Negatively regulating TLR4/NF$\mathrm{kB}$ signaling via PPAR in endotoxin-induced uveitis. Biochim Biophys Acta-Mol Basis Dis. 2014;1842(7):1109-20.

35. Ronald DS, Huang HS. Corneal Penetration Behavior of $\beta$-Blocking Agents I: Physicochemical Factors. J Pharm Sci. 1983;72(11):1266-72.

36. Vasconcelos A, Vega E, Pérez Y, Gómara MJ, García ML, Haro I. Conjugation of cell-penetrating peptides with poly(Lactic-co-glycolic acid)-polyethylene glycol nanoparticles improves ocular drug delivery. Int J Nanomedicine. 2015;10:609- 
31.

37. Arechabala B, Coiffard C, Rivalland P, Coiffard LJM, De Roeck-Holtzhauer Y. Comparison of cytotoxicity of various surfactants tested on normal human fibroblast cultures using the neutral red test, MTT assay and LDH release. J Appl Toxicol. 1999;19(3):163-5.

38. Jiang Y, Thakran S, Bheemreddy R, Ye EA, He H, Walker RJ, Steinle JJ. Pioglitazone normalizes insulin signaling in the diabetic rat retina through reduction in tumor necrosis factor and suppressor of cytokine signaling $3 *$. J Biol Chem. 2014;289(38):26395-405.

39. Murata T, Hata Y, Ishibashi T, Kim S, Hsueh WA, Law RE, Hinton DR. Response of experimental retinal neovascularization to thiazolidinediones. Arch Ophthalmol. 2001;119(5):709-17. 


\section{Legend to Figures}

Fig.1: Surface response of PGZ-NSs at $9.5 \mathrm{mg} / \mathrm{ml}$ PLGA-PEG concentration. (A) PGZ and Tw 80 concentrations influence on the NSs ZP. (B) PGZ and Tw 80 concentrations influence on the NSs EE.

Fig. 2: Transmission electron microphotograph of the PGZ-NSs.

Fig. 3. Interactions studies. (A) X-ray diffraction patterns. (B) Differential scanning calorimetry.

Fig. 4: Biopharmaceutical behavior. (A) In vitro release profile of PGZ-NSs and Free PGZ. (B) Transcorneal permeation. (C) Transcleral permeation.

Parameters values are expressed as average $\pm \mathrm{SD} ; * * * * p<0.0001$.

Fig. 5.: Cell viability of Y 79 cells exposed to PGZ-NSs, Free PGZ and Tw $802 \%$ at different concentrations.

Fig. 6.: Comparison of anti-inflammatory efficacy after SA-induced inflammation in the pig's eye.

Values are expressed as average $\pm \mathrm{SD} ; * * * p=0.0008 * * * * p<0.0001$ significantly lower than the inflammatory effect induced by SA. 